Estimating the Relative Efficiency

of Various Forms of Prevention

at Different Stages of a Drug Epidemic

D.WINKLER, J.P. CAULKINS,

D.A. BEHRENS, G.TRAGLER

Working Paper No. 264

January 2002 



\title{
Estimating the Relative Efficiency of Various Forms of Prevention At Different Stages of a Drug Epidemic
}

\author{
Doris Winkler ${ }^{1}$, Jonathan P. Caulkins ${ }^{2,3}$, Doris A. Behrens ${ }^{1,4 ⿴ 囗 6}$ \\ and Gernot Tragler ${ }^{1}$
} ${ }^{1}$ Department of Operations Research and Systems Theory, Vienna University of Technology, Argentinier-
strasse 8, A-1040 Vienna, Austria
${ }^{2}$ H. John Heinz III School of Public Policy and Management, Carnegie Mellon University, 5000 Forbes
Ave., Pittsburgh, PA 15213, U.S.A.
${ }^{3}$ RAND, 201 N. Craig St., Suite 102, Pittsburgh, PA 15213, U.S.A.
${ }^{4}$ Department of Economics, University of Klagenfurt, Universitaetsstrasse 65-67, A-9020 Klagenfurt, Aus-
tria

\begin{abstract}
Drug use and problems change dramatically over time in ways that are often described as reflecting an "epidemic cycle". We use simulation of a model of drug epidemics to investigate how the relative effectiveness of different types of prevention varies over the course of such an epidemic. Specifically we use the so-called LHY model (see Behrens et al., 2000b) which includes both "contagious" spread of initiation (a positive feedback) and memory of past use (a negative feedback), which dampens initiation and, hence, future use. The analysis confirms the common sense intuition that prevention is more highly leveraged early in an epidemic, although the extent to which this is true in this model is striking, particularly for campaigns designed to leverage awareness of the drug's dangers. The findings also suggest that the design of "secondary" prevention programs should change over the course of an epidemic.
\end{abstract}

Keywords: Illicit drugs, memory, demand side control, prevention, dynamical systems, simulation, reputation

\footnotetext{
+ This research was partly financed by the Austrian Science Foundation under contract No. P14060OEK.

* Corresponding author: Department of Operations Research and Systems Theory, Vienna University of Technology,Argentinierstrasse 8,A-1040Vienna,Austria,dbehrens@eos.tuwien.ac.at
} 



\section{Introduction}

This paper uses simulations of mathematical models of drug use to estimate the relative effectiveness of different types of prevention over the course of a drug epidemic. There is widespread popular support for prevention generally as a means of controlling illicit drug use and other criminal behavior (Maguire and Pastore, 1996, pp.168-169), but there are many kinds of prevention. Evidence is beginning to accumulate concerning the relative effectiveness of different strategies for preventing crime and violence. Greenwood et al. (1998), for example, synthesized the literature comparing the cost-effectiveness of such diverse interventions as prenatal visits, early daycare, parent training, high-school graduation incentives, and supervision for juvenile delinquents for reducing criminal activity. Aos et al. (2001) and Caulkins et al. (forthcoming) do the same for an even broader array of interventions.

Comparable evidence is not yet available for the different types of drug prevention. School-based primary prevention programs (such as DARE, ALERT, and Life Skills) have been studied individually (Tobler, 1997; Ellickson et al., 1993; and Botvin et al., 1995) and compared as a broad class to drug treatment, enforcement, and interdiction (Caulkins et al., 1999). There is not a comparable empirical literature rigorously evaluating secondary prevention (which seeks to moderate use by people who have already initiated but who are not yet addicted) or campaigns designed to raise awareness of the risks of drug use. This does not, and perhaps should not, deter investment in these other forms of prevention. E.g., mass media advertising was a centerpiece of the Clinton administration's drug prevention strategy (ONDCP, 1998). It does, however, preclude systematic comparison of the absolute cost-effectiveness of these three broad types of prevention.

We can, however, still make one very important comparison. There is increasing evidence that the effectiveness of different types of interventions, including prevention, can vary dramatically over the course of a drug epidemic (Caulkins et al., 1999; Behrens et al., 2000a; Tragler et al., 2001). This should not be surprising because drug use spreads initially through a positive feedback loop (current users promote initiation of new users) that is eventually overwhelmed by a lagged, negative feedback (e.g., the drug develops a negative reputation which suppresses initiation). By including those feedback loops in a model of flows between various types or stocks of use, we can explore how making a given change to a flow at different points in the epidemic will affect the total future demand for the drug. We still will not know how much it costs to "purchase" that temporary change in the flow, but we will have an estimate of how much it would be worth to do so. Or, looking at it another way, we can identify the flows and points in time for which small changes yield big impacts on drug use in the long run. Fur- 
thermore, if the cost of purchasing any given change is roughly the same from year to year, we will get a sense of how the cost-effectiveness of making that type of change might vary over the course of the epidemic.

\section{A Brief Sketch of the $L H Y$ Model}

Looking across patterns of use over time for different drugs in different countries and in different eras, a few simple observations can be made (Caulkins, forthcoming). First, drug use can increase very rapidly from initially low levels. The increases are far faster and more dramatic than corresponding changes in underlying social variables (e.g., unemployment). We take this as evidence of important internal dynamics, specifically a positive feedback loop. Indeed, there is widespread agreement among experts thatinitiation is driven substantially by current users introducing novices to the drug.

Second, this rapid spread eventually peaks, and initiation subsequently declines, sometimes dramatically, sometimes more modestly. There are two broad classes of epidemic models that account for this behavior. One, drawing directly on models of pathogenic epidemics, suggests that the spike in initiation is attributable to the drug spreading through a pool of "susceptibles" who had not previously been exposed to the drug. (See, e.g., Rossi and Schinaia, 1998.) That may in fact be part of the story, but we prefer a second explanation, advanced by Musto (1973).

Musto (1973) suggests that over time some initiates progress to heavy or dependent use. Their problems then become a sort of negative advertisement for the drug, suppressing initiation. Kleiman (1992) elaborates this idea by pointing out that it is not just the absolute number of people who suffer adverse consequences that matters, but the relative proportion. A hundred sorry addicts buried in a sea of millions of happy recent initiates paints a different image than would just the addicts alone. Musto adds one caution. Memories and negative reputations are not infinitely lived. When the current generation of youth no longer remembers the problems their forebears experienced with the substance, they become vulnerable to a new wave of drug use, which may explain why the epidemics of cocaine and opiate use around the turn of the last century were separated from the more recent epidemics which began in the late 1960s and 1970s by several decades of very low rates of use.

\footnotetext{
${ }^{1}$ Kaplan (1983) gives a clear explanation for why drug "pushers" pushing drugs on unsuspecting innocents is not the source of most initiation, old myths notwithstanding.
} 
Musto's and Kleiman's insights have been converted into formal mathematical models for US cocaine use (Everingham and Rydell, 1994; Behrens et al., 1999), culminating in the so-called LHY model (Behrens et al., 2000b). We use this model as the basis for our simulations of prevention's effectiveness at different points over the course of an epidemic.

The $L H Y$ model depicted in Figure 1 is a continuous-time dynamic model of drug demand that differentiates between light $(L(t))$ and heavy $(H(t))$ users, with parameters $a, b$, and $g$ representing the annual rate at which light users quit, light users escalate to heavy use, and heavy users quit, respectively. The third state represents the decaying memory of heavy user years (denoted $Y(t)$ ). When the outflow rate or the "memory parameter" $\delta$ is small, memories of drug abuse are long-lived; when $\delta$ is large, memory of heavy users' years of use and associated problems dissipates rapidly.

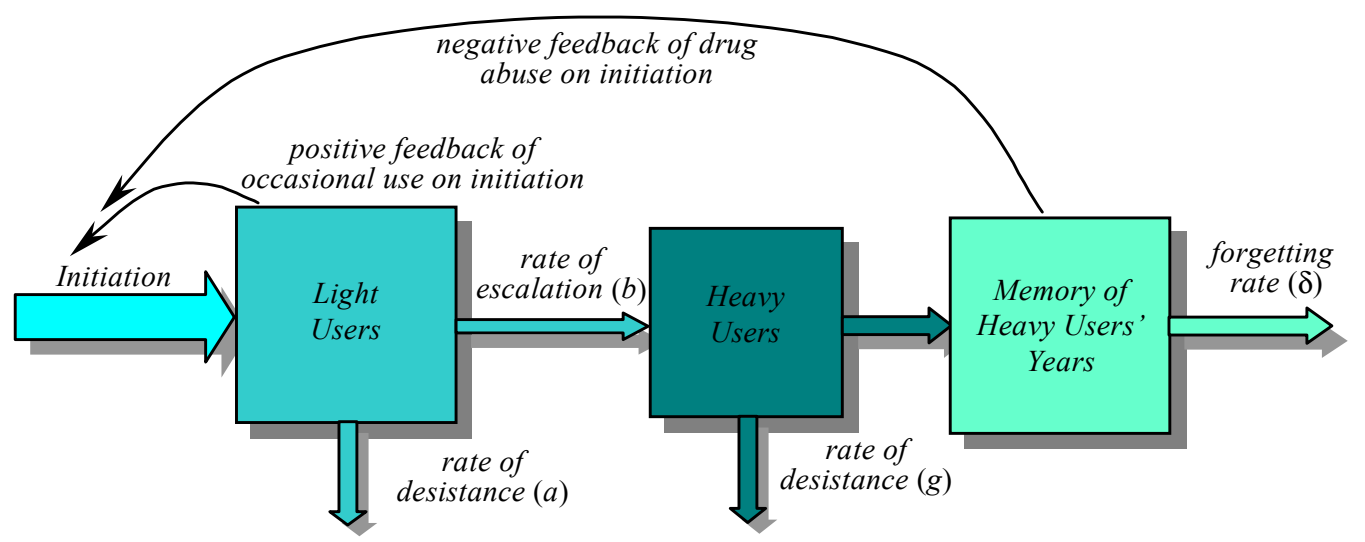

Figure 1: Flow chart of the $L H Y$ model (cf. Behrens et al., 2000b)

Omitting the explicit denotation of time this leads to the following descriptive three-state model of light $(L)$ and heavy $(H)$ use and heavy user years $(Y)$ :

$$
\begin{array}{rlrl}
\dot{L} & =I(L, Y)-(a+b) L, & L(0)=L_{0}, \\
\dot{H} & =b L-g H, & H(0)=H_{0}, \\
\dot{Y} & =H-\delta Y, \quad Y(0)=Y_{0}, \\
L & =\text { number of light users, } \\
H & =\text { number of heavy users, } \\
Y & =\text { decaying heavy user years, } \\
I & =\text { initiation, }
\end{array}
$$


$a=0.163$ annual rate at which light users quit,

$b=0.024$ annual rate at which light users escalate to heavy use,

$g=0.062$ annual rate at which heavy users quit,

$\delta=0.291$ forgetting rate.

The memory's ( $Y$ 's) dampening of contagious initiation has been modeled as a negative exponential function of the memory of the years of drug abuse relative to the number of current light users. This functional form seems to fit the (limited) available data through the peak of the epidemic, although other forms do as well. (For details see Behrens et al., 1999, 2000b.) As in many marketing diffusion models (see, e.g., Bass, 1969), the endogenous initiation function includes some "spontaneous" initiation (e.g., due to immigration or "innovators"), but most occurs through interaction with current light users. It has been specified by

$$
I(L, Y)=\tau+s L \operatorname{Exp}\left(-q \frac{Y}{L}\right)
$$

$\tau=5 \times 10^{4} \quad$ number of innovators per year,

$s=0.610$ annual rate at which light users attract non-users,

$q=3.443$ constant which measures the deterrent effect of heavy use.

There is some evidence that the negative exponential overstates the reputation feedback in the 1990s. In particular, the amount of endogenous initiation per light user seemed to reach a floor of 0.1 (and may even have ticked back up recently). To be conservative with respect to a central conclusion, namely that prevention is more effective earlier than later in the epidemic, the initiation function is modified to reflect this empirically observed floor:

$$
I(L, Y)=\tau+L \operatorname{Max}\left\{0.1, s \operatorname{Exp}\left(-q \frac{Y}{L}\right)\right\} .
$$

The parameter values are based on the current US cocaine epidemic and are taken from Behrens et al. (2000b).

\section{Simulation of the Effectiveness of Various Forms of Prevention}

We are interested in three categories of prevention: primary prevention, secondary prevention, and awareness campaigns. (See Table 1.) 
Table 1: Categories of prevention

\begin{tabular}{lll}
\hline Type of Prevention & Definition & Interpretation in the Model \\
\hline Primary Prevention & $\begin{array}{l}\text { Preventing people from initi- } \\
\text { ating drug use. }\end{array}$ & $\begin{array}{l}\text { Reducing the flow into light use } \\
(L) \text {, i.e., reducing initiation. }\end{array}$ \\
Secondary Prevention & $\begin{array}{l}\text { Moderating the use of people } \\
\text { who have already initiated but } \\
\text { not yet become dependent. }\end{array}$ & $\begin{array}{l}\text { Reducing escalation from light to } \\
\text { have use (flow } b) \text { and/or increasing } \\
\text { quitting from light use (flow } a) .\end{array}$ \\
Awareness campaigns & $\begin{array}{l}\text { Promoting awareness of the } \\
\text { drug's harmfulness }\end{array}$ & $\begin{array}{l}\text { Increasing the impact of the stock } Y \\
\text { or reducing the rate at which } Y \\
\text { decays. }\end{array}$ \\
\hline
\end{tabular}

For each category we can investigate separately two facets or components. For primary prevention we can think about interventions that reduce initiation by a certain percentage or by a certain number of individuals. School-based education is customarily thought of as affecting a certain percentage of potential initiates, in part because it is usually given to all students so the number of students affected is proportional to the number who were going to start using. Other programs (e.g., mentoring programs) work one-on-one with individual students. The number of students "saved" depends on the number enrolled in the program, not the number who were going to start using, as long as enough were going to start that the program is not "squandered" on people who were not at risk in the first place.

For secondary prevention we can distinguish between preventing light users from escalating to heavy use (reducing flow $b$ ) and encouraging them to cease use altogether (increasing flow $a$ ). That is not to say that there are necessarily separate programs that each affect only flow $a$ or flow $b$. Rather, we are distinguishing two mechanisms of influence through which a single program may act, although it may be possible to design programs that put relatively greater emphasis on one or the other.

Awareness campaigns could be construed as amplifying the impact of a given memory of negative consequences (increasing $q$ ) and/or as helping to preserve those memories (reducing $\delta$ ). Again, the image is not so much that there are separate programs that have these distinct impacts. Rather, these are two possible mechanisms through which an awareness campaign could act, but one can imagine designing campaigns to give relatively greater emphasis to one or the other.

\footnotetext{
2 "Squander" in quotes because prevention programs generally, including mentoring, generate a wide range of benefits, not just benefits associated with reduced drug use.
} 
For five of these six prevention mechanisms we model an enhanced prevention effort as modifying the corresponding parameter by a certain percentage. We model the other, specifically the second type of primary prevention, as reducing initiation by a certain constant.

For each mechanism and for each year (1970-2020) we determined how big a change needed to be maintained for one year in order to reduce the total future discounted, weighted number of user-years by a certain amount. I.e., we asked how big a change was needed to reduce

$$
J=\int_{0}^{\infty} e^{-r t}(L(t)+7.25 H(t)) d t
$$

by a certain amount subject to model (1) and (2'). Heavy users are weighted 7.25 times as heavily as light users in the objective because heavy users consume about 7.25 times as much per user per year (Everingham and Rydell, 1994). Following the related literature, we discount at an annual real rate of $r=0.04$. For ease of exposition, we will refer to this objective of future discounted weighted user-years simply as future discounted demand.

The amount by which this objective is reduced is arbitrary as long as it is the same for each prevention mechanism and small enough that it is not substantially affected by diminishing returns or other non-linear effects. We chose as a target the number of weighted user-years that would have been associated with \$10 million in social costs under 1992 conditions.

Initial values $\left(L_{0}, H_{0}\right.$, and $\left.Y_{0}\right)$ come from the numerically calculated drug epidemic induced by the LHY model as depicted by Figure 2. (Behrens et al., 2000b, show that the fit with historical data is quite good to date.) Since all calculations are done numerically - not analytically - we use a finite (500-year) approximation to an infinite planning horizon. Truncating after 500 years is not a concern because of the discounting and because the epidemic approaches a steady state well before then.

All calculations were done using MATHEMATICA 4.1 (Wolfram, 1996) and MATLAB 5.2.0.3084 (registered trademark of MathWorks Inc.) under Windows 98 operating system.

\footnotetext{
${ }^{3}$ Everingham and Rydell (1994) report annual consumption of 16.42 and 118.93 grams of cocaine for light and heavy users in 1992, respectively, and Behrens et al. (2000a) ascribe a social cost of $\$ 113$ per gram. Newer evidence suggests the social cost per gram may be even higher, but that has no effect on these calculations because the size of the reduction in the objective (3) is unimportant as long as it is consistent across programs.
} 


\section{Results}

\subsection{Identifying "High Leverage" Stages for Prevention}

Figure 2 shows the epidemic cycle, as modeled by the $L H Y$ model. Initiation peaks in the late 1970s, followed by peaks in the number of light users $(1982 / 83)$, total number of users (1983, not shown), heavy users (1992), and the memory of years of abuse (1996). The first question to ask is, at what points in this epidemic cycle are the various prevention mechanisms most highly "leveraged" in the sense that only small changes in the corresponding flow parameter are required to reduce discounted future demand by a given amount?

The bars below the line graph in Figure 2 show the answer. For all but the second type of primary prevention, dark shading indicates years for which changes of less than $0.025 \%$ were sufficient to produce our target reduction in demand. Moderate (light) shading indicates years for which the required changes were between $0.025 \%-0.1 \%(0.1 \%-0.4 \%)$. Years outside the bars altogether required changes of more than $0.4 \%$.

The second type of primary prevention involves reducing initiation by a certain number of individuals, not a certain percentage. For it the dark bar corresponds to one-year reductions in initiation of less than 100 individuals, and moderate (light) bars indicate reductions of 100-400 (400-1600) individuals. ${ }^{4}$ Years outside the bars required initiation reductions of more than 1600 individuals to achieve the target reduction in discounted future demand.

For sake of comparison, we also consider as a foil increasing the exit rate for heavy users. This intervention is labeled treatment, although heavy users could also be removed through incarceration.

Note that primary prevention programs are usually conducted with youth (e.g., 13-14 year olds), whereas cocaine initiation is most common in young adulthood (median age 21). Hence, there is a lag between program implementation and effect. Below, descriptions of the timing refer to the timing of the effects, not the implementation. For secondary prevention and information campaigns, implementation and effect are essentially contemporaneous, but for primary prevention it should be understood that the implementation precedes effect by on the order of eight years.

Prevention in early years is generally more highly leveraged than in later years. Table 2 underscores this point by listing the years in which each intervention could achieve the target reduction in future demand with the smallest possible parameter change. The exception is secondary prevention that reduces escalation to heavy use. Early in the epidemic, reducing progression to heavy use is actually counter-

\footnotetext{
${ }^{4}$ These correspond to the stated percentages when interpreted as the percentage of initiation estimated to have occurred in 1972 by Johnson et al. (1996).
} 
Table 2: Smallest parameter changes (and associated years) leading to a $\$ 10$ million reduction in social costs

\begin{tabular}{lllcc}
\hline Type of prevention & & $\begin{array}{l}\text { Smallest } \\
\text { parameter change }\end{array}$ & $\begin{array}{c}\text { Year in } \\
\text { which } \\
\text { change is } \\
\text { smallest }\end{array}$ & $\begin{array}{c}\text { Parameter } \\
\text { change in "base } \\
\text { year" 1985 }\end{array}$ \\
\hline 1. Primary & $I \rightarrow I(1-\Psi)$ & $\Psi=1.415 \times 10^{-5}$ & 1970 & $0.06 \%$ \\
2. Individual level primary & $I \rightarrow I-\Phi$ & $\Phi=10.691$ & 1970 & $\approx 468$ \\
3. Secondary & $a \rightarrow a(1+\alpha)$ & $\alpha=3.973 \times 10^{-5}$ & 1971 & $0.04 \%$ \\
4. Secondary & $b \rightarrow b(1-\beta)$ & $\beta=1.158 \times 10^{-3}$ & 1984 & $0.11 \%$ \\
5. Information campaign & $q \rightarrow q(1+\eta)$ & $\eta=3.589 \times 10^{-5}$ & 1973 & $0.21 \%$ \\
6. Memory campaign & $\delta \rightarrow \delta(1-\zeta)$ & $\zeta=7.223 \times 10^{-5}$ & 1972 & $9.55 \%$ \\
\hline Treatment & $g \rightarrow g(1+\gamma)$ & $\gamma=1.510 \times 10^{-3}$ & 1986 & $0.15 \%$ \\
\hline
\end{tabular}

productive because it delays the accumulation of a negative reputation, which ultimately is a more powerful force for reducing use than is the prevention program itself. Later in the epidemic, specifically past the peak in light use, the negative reputation is already well established and preventing escalation does not have this perverse side-effect. ${ }^{5}$ In this model, treatment early in the epidemic can have a similar perverse effect, but the range of years for which it is highly leverage is broader. In both cases, the baseline flows are low $(b=0.024 ; g=0.062)$ so larger percentage changes in these flows are needed to have a significant effect.

Conversely, once the negative reputation is well established, awareness campaigns become relatively superfluous. In this model, they are extremely highly leverage early in the epidemic, but redundant later. Another way to look at leveraging is to ask, if one could reach down in any year and remove one light user (equivalent to preventing one initiation) or one heavy user, which year should one choose? Figure 3 shows the relative reduction in future discounted demand generated by removing one light or one heavy user, by year from 1978-2020. The figure shows clearly that preventing an initiation early in an epidemic is worth more, in terms of reduced future demand, than is preventing an initiation later in the epidemic, after the contagious spread stage has passed. Indeed, the figure is plotted for 1978-2020 not 1970-2020 because the benefit of preventing an initiation in

\footnotetext{
${ }^{5}$ Note: In a sense, this intervention is also most highly "leveraged" in the initial phase of the epidemic, i.e., in 1970. It is just leveraged in the wrong direction! 1984 is the year in which the smallest positive parameter change achieves the target change in future demand. The (negative) change needed in 1970 is actually smaller in absolute value.
} 


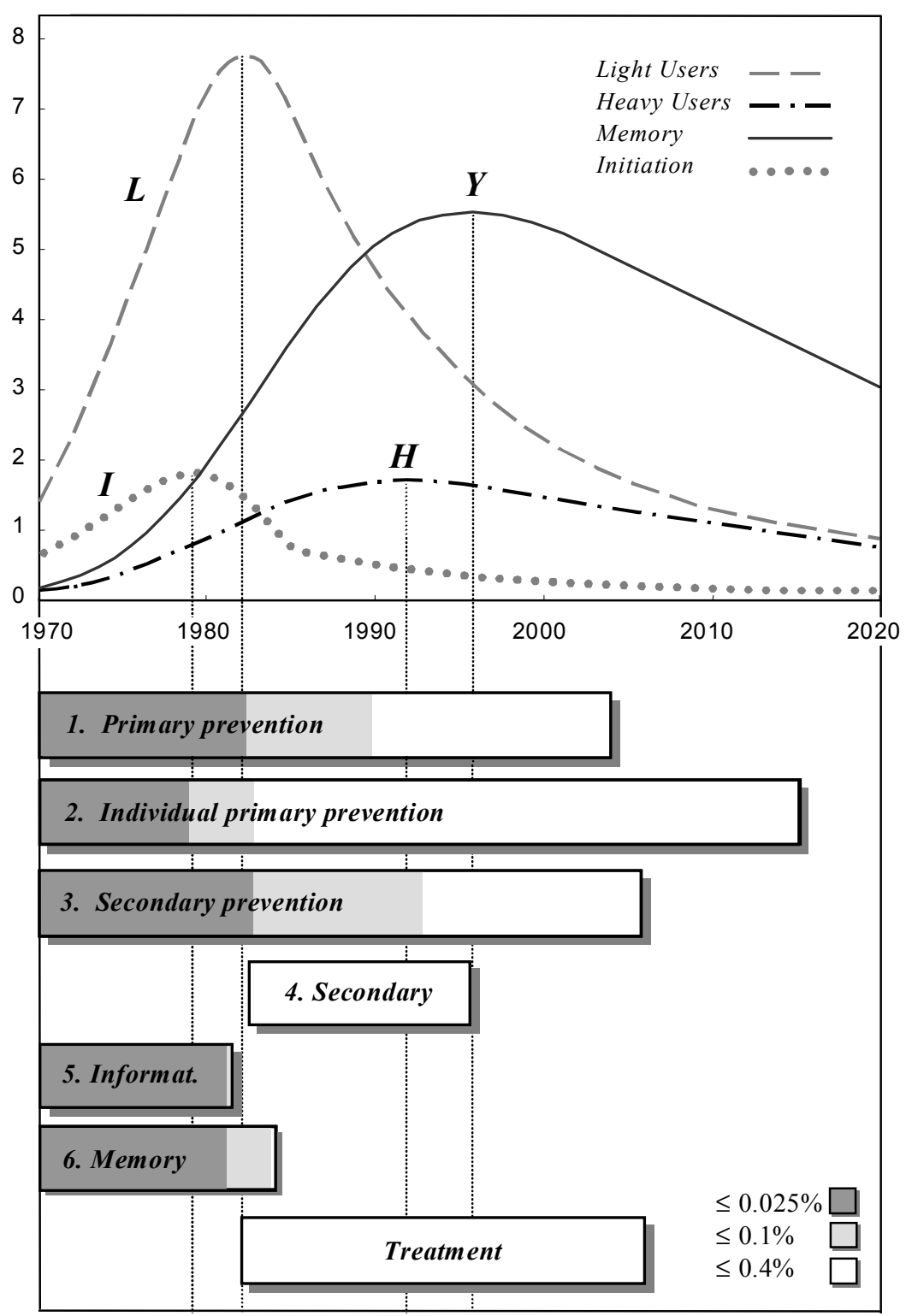

Figure 2. Plot of modeled initiation, numbers of users, memory of abuse in millions (cf. Behrens et al., 2000b) and periods where prevention is most leveraged (parameter changes of less than $x \%$ yield a pre-specified reduction in future discounted demand). Initial conditions for $L H Y$ system are determined by $L_{1970}=1,400,000$, $H_{1970}=130,000, Y_{1970}=110,000$. 
1970 is about 7.5 times that in 1978. Extending the figure back to 1970 makes it hard to see the trends in the relative benefits of eliminating one heavy user.

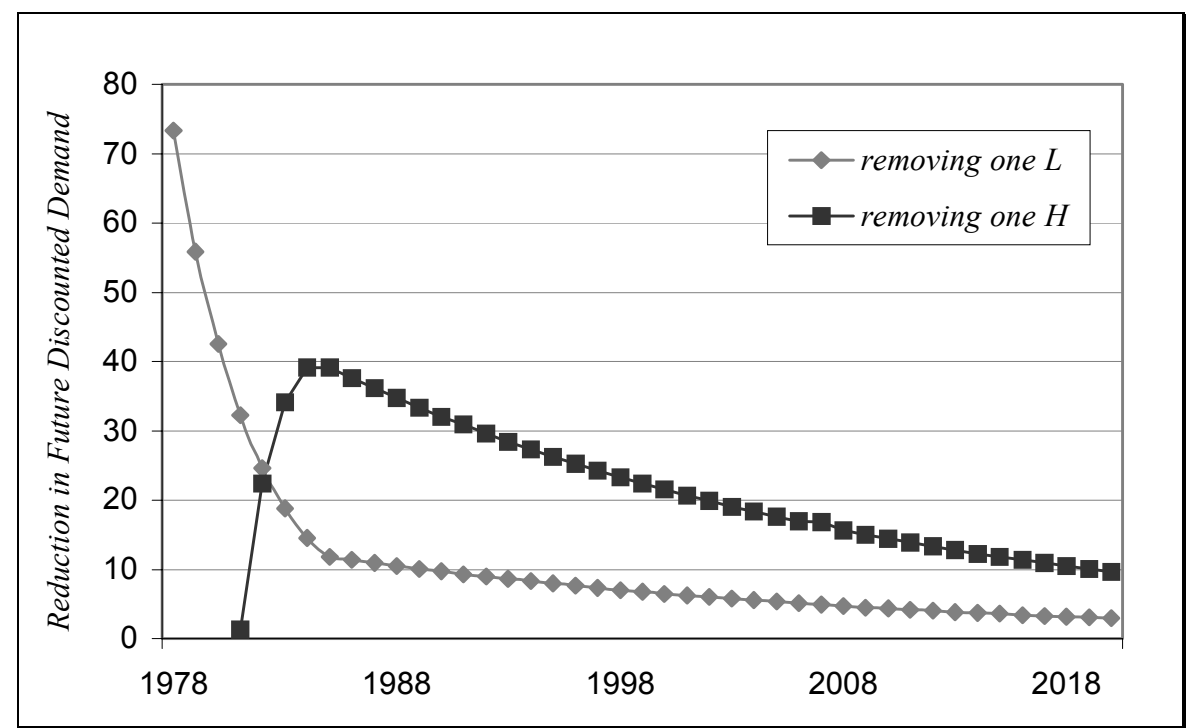

Figure 3. Reduction in future discounted demand generated by removing one light user in year $x$ relative to 1970 and by removing one heavy user in year $x$ relative to 1986; $x$ from 1970-2020.

The LHY model (1) and (2') estimates that preventing a single initiation in 1970 would have averted an amount of subsequent demand that in 1992 was associated with social costs of about $\$ 0.9$ million. The $\$ 0.9$ million figure should not be taken too seriously because the social cost per unit of demand likely varied over time, and 1970 is at the edge of when the model can plausibly be expected to reflect the epidemic dynamics. Nevertheless, since school-based prevention only consumes about $\$ 150$ worth of resources per youth (Caulkins et al., 1999), it is clear that prevention does not have to be very effective in absolute terms to be cost-effective when conducted before or during the explosive, contagious spread of a drug such as cocaine.

The figure shows that removing a heavy user is most beneficial around the peak in heavy use and with this model is actually counter-productive before the peak in light use is observed (for reasons already discussed). By 1991, the ratio of the benefit of removing a heavy vs. a light user has stabilized at about 3.3:1.

\footnotetext{
${ }^{6}$ This 3.3:1 ratio stems from the facts that residual time in the heavy use state is about triple that in the light use state, heavy users consume at a rate that is 7.25 times higher, light users have a roughly one in
} 
We can do a similar calculation for all of the types of prevention. We calculate the reduction in future demand caused by changing a parameter $(\Psi, \Phi, \alpha, \beta, \eta, \zeta, \gamma)$ by a certain, fixed amount for one year, and we do this calculation for each year and for each type of prevention.

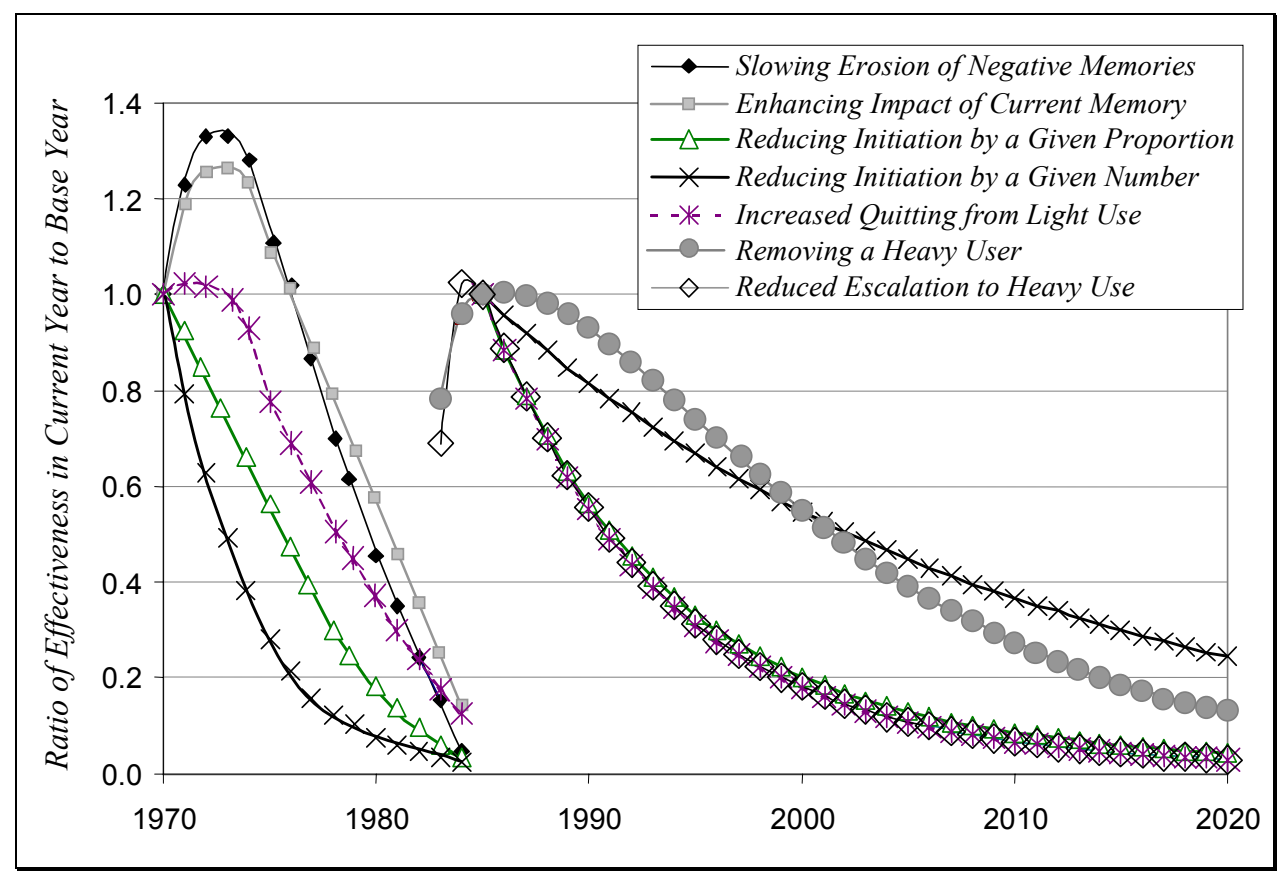

Figure 4. Variation over time in effectiveness (scales to 1 in 1970 on the left and 1985 on the right).

Then in Figure 4 we scale the results for each prevention type so they all pass through $100 \%$ in 1970 and 1985 on the left and right sides of the figure, respectively. The effectiveness of the awareness campaigns plummets in the late 1970 s, to the point that they are not even plotted on the right hand side of Figure 4. Conversely, reducing escalation and removing heavy users only appear on the right hand side. The lines for reducing initiation by a given proportion and increasing quitting from light use track each other, and differ from the line for reducing use by a given number, which generally shows the slowest decay after initiation peaks in the late 1970s.

eight chance of escalating to heavy use, and at the later stages of the epidemic light users stimulate about 0.1 initiations per year over the average 5.3 year career of light use, after adjusting for discounting and rounding. 


\subsection{Sensitivity Analysis with Respect to the Drug's Initial Reputation}

So far we have done all our investigations as if we could travel back in time to the early years of the current US cocaine epidemic. The next epidemic could be different on a variety of dimensions, so many forms of sensitivity analysis could potentially be relevant. But for practical purposes sensitivity analysis with respect to the initial reputation level stands out as being of particular interest for several reasons. First, we have less information about the initial reputation level than we do about the initial use levels. Second, if a renewed cocaine epidemic threatened in the near or medium term future, it is hard to imagine that cocaine's reputation at the onset of that epidemic would not be more negative than what we presume it was in 1970.

We computed the discounted social cost averted associated with reducing initiation by a given proportion - sufficient to save $\$ 10$ million if the intervention is performed in $1992(\psi=0.0218)$ - as a function of the initial number of light and heavy users, $L_{0}$ and $H_{0}$, for many different values of $Y_{0}$. We find that the benefits of traditional prevention are decreasing in $Y_{0}$. I.e., a negative reputation such as from memory of past heavy use is a substitute for prevention. If there is "a lot" of memory (roughly 5 times the current level of heavy users) then the value of prevention is not greatly affected by the current number of light users (presumably because there will be no epidemic spread of use). Thus the severity of the epidemic and, hence, the value of prevention can be very different for two drugs that are identical in all respects except their initial reputation levels. In particular, drugs which (inappropriately) have a more benign reputation pose a bigger threat of a severe epidemic.

\subsection{Sensitivity Analysis with Respect to the Functional Form of the Drug's Reputation}

The evolution of the modeled epidemic depends nontrivially on how exactly reputation feeds back to suppress initiation. Hence we not only conducted sensitivity analysis with respect to specific parameter values, but also with respect to the functional form for this feedback. In particular, analyses were repeated with two other functional forms: the original initiation function used in past work that did not have the "floor" (Equation (2)) and a floor on initiation rather than "infectivity", specifically

$$
I(L, Y)=\tau+\operatorname{Max}\left\{100,000, s L \operatorname{Exp}\left(-q \frac{Y}{L}\right)\right\} .
$$


With initiation modeled by Equation (2), the main difference is that no variant of primary prevention or awareness campaigns is particularly valuable after the early 1990s because initiation becomes very modest even in the absence of such measures. Thus the drop off in effectiveness over time is even steeper with initiation as modeled by Equation (2) than is described above. The effectiveness of reducing escalation and treatment are less affected, so their effectiveness rises relative to that of the other interventions between the mid 1980s and the late 1990s (secondary prevention) and into the $21^{\text {st }}$ century (for treatment). E.g., late in the epidemic removing a heavy user is about six times as valuable as removing a light user, not just 3.3 times as effective, because late in the epidemic light users recruit fewer new initiates when initiation is modeled by Equation (2).

With initiation modeled by Equation (2') results are intermediate but closer to those with Equation (2) because the floor on initiation does not take effect until relatively late. That is, the central findings are robust with respect to these variations in the modeling of initiation.

\section{Conclusions}

The central finding of this paper is that the effectiveness of various types of prevention varies substantially over the course of a drug epidemic such as the recent US cocaine epidemic. Furthermore the extent of this variation differs across types of prevention, so the effectiveness of each type of prevention relative to the effectiveness of the other types also varies. Hence, one would expect that in an optimal drug control strategy, not only would the level of prevention funding vary over time, but so would the mix of prevention strategies funded.

We considered three classes of prevention campaigns: primary prevention that seeks to prevent initiation, secondary prevention that moderates the use of "light" users who have already initiated, and awareness campaigns that enhance the deterrence of initiation by societal memories of adverse consequences of "heavy" use. Within each class we considered two types of prevention.

Awareness campaigns' comparative advantage comes early in an epidemic. Indeed, the decline in effectiveness is so extreme that they seem to have almost no role after the peak in initiation, when the genuine consequences of heavy use are sufficient to establish a negative reputation. In this model, these findings hold with equal force whether the effect of the awareness campaign is to enhance the impact of current memories or to slow the erosion of memories over time.

Primary prevention is also more highly leveraged early in the epidemic, but continues to be useful well past the peak in initiation. This is particularly true for primary prevention programs that affect a certain number of individuals per dollar 
spent (e.g., through individualized programs like mentoring) rather than programs that affect a certain proportion of a cohort (e.g., through school-based education programs in which all students participate).

This distinction is a direct consequence of the way drug epidemics evolve. To see this, compare prevention in 1971 and 1976. With the present model, preventing a person from initiating was about 3.5 times as valuable in 1971 as in 1976, and Johnson et al. (1996) report that initiation increased by a factor of 3.5 over those five years. Hence there would be no change over that period in the benefit of primary prevention that reduces initiation by a given percentage. Such programs would affect 3.5 times as many youth in 1976 (because baseline initiation rates were higher), but the value per youth is reduced, leaving no change in the value of the program. In contrast, a prevention program that prevented a fixed number of youth from initiating would have lost $70 \%$ of its value between 1971 and 1976 . While initiation rates are rising (through about 1980 for the US cocaine epidemic), the first type of primary prevention loses effectiveness more slowly than does the second. After initiation has peaked, the opposite is true.

We distinguished two effects of secondary prevention: encouraging light users to quit and discouraging them from escalating to heavy use. The former behaves like primary prevention, specifically programs that reduce initiation by a given proportion. Effectiveness is greatest early in the epidemic and declines over time, but much less precipitously than is the case for awareness campaigns.

The time profile of effectiveness for preventing escalation is entirely different. With the current model, early in an epidemic the marginal impact of an additional light user is very bad whereas the marginal impact of a heavy user is actually positive. This seemingly perverse result occurs because there is a positive feedback from light use to initiation (into light use) and a negative feedback from heavy use to initiation (through the accumulated memory of the adverse consequences of heavy use). So preventing escalation prevents someone from moving out of the role of promoting initiation (by introducing friends to drug use) and into the role of dampening initiation (by "advertising" the dangers of drug use). Of course preventing escalation also has the immediate beneficial effect of preventing that individual's rate of consumption from increasing. Late in the epidemic, when the drug's negative reputation is established and contagious spread has ended, this direct effect on the individual's own use swamps the indirect effects on use by others, but not so early in the epidemic.

With the current parameter values, preventing escalation is counterproductive before the peak in light use. Fairly shortly thereafter it becomes productive and has its maximum impact. That impact then subsequently decays roughly at the rate effectiveness decays for primary prevention. The effectiveness 
of removing heavy users (e.g., through treatment) varies over time in a similar manner.

All of these statements pertain to changes in the value of making a given change to a flow, e.g., initiation. This paper is silent on how the cost of "purchasing" such a change might vary over time. Hence, the results pertain only to effectiveness, not cost-effectiveness.

The results seem qualitatively robust, but of course the specific numbers depend on modeling assumptions pertaining to both parameter values and model structure (e.g., the functional form used to describe how a negative reputation dampens initiation). Replication with epidemic models based on other drugs and/or epidemics in different countries would be instructive. 


\section{References}

[Aos, et al., 2001] S. Aos, P. Phipps, R. Barnoski, and R. Lieb: The Comparative Costs and Benefits of Programs to Reduce Crime, Version 4.0, 01-05-1201, Washington State Institute for Public Policy: Olympia, WA, 2001.

[Bass, 1969] F.M. Bass: "A new product growth model for consumer durables". Management Science, 15 (1969): 215-227.

[Behrens et al., 1999] D.A. Behrens, J.P. Caulkins, G. Tragler, J.L. Haunschmied, and G. Feichtinger: "A Dynamic Model of Drug Initiation: Implications for Treatment and Drug Control”. Mathematical Biosciences, 159 (1999): 1-20.

[Behrens et al., 2000a] D.A. Behrens, J.P. Caulkins, G. Tragler, and G. Feichtinger: "Optimal Control of Drug Epidemics: Prevent and Treat - But Not At the Same Time?". Management Science, 46(3) (2000): 333-347.

[Behrens et al., 2000b] D.A. Behrens, J.P. Caulkins, G. Tragler, G. Feichtinger: "Memory, Contagion, and Capture Rates: Characterizing the Types of Addictive Behavior that Are Prone to Repeated Epidemics". Discussion Paper No 251 of the Institute of Econometrics, OR, and Systems Theory, Vienna University of Technology: Vienna, Austria, 2000.

[Botvin et al., 1995] G.J. Botvin, E. Baker, L. Dusenbury, E.M. Botvin, and T. Diaz: "Long-Term Follow-Up Results of a Randomized Drug Abuse Prevention Trial in a White Middle-Class Population". Journal of the American Medical Association, 273 (1995): 1106-1112.

[Caulkins et al., 1999] J.P. Caulkins, C.P. Rydell, S.S. Everingham, J. Chiesa, and S. Bushway: "An Ounce of Prevention, a Pound of Uncertainty: The CostEffectiveness of School-Based Drug Prevention Program". MR-923-RWJ, RAND: Santa Monica, CA, 1999.

[Caulkins et al., forthcoming] J.P. Caulkins, P.W. Greenwood, J. Chiesa, A. Geller: Review of Lessons from Uncertainty: Assembling Crime Prevention Strategies from Cost-Effectiveness Estimates. DRR-2597-WTGF, RAND: Santa Monica, CA, forthcoming.

[Ellickson et al., 1993] P.L. Ellickson, R.M. Bell, and K. McGuigan: Preventing Adolescent Drug Use: Long-Term Results of a Junior High Program. American Journal of Public Health, 83(6) (1993): 856-861.

[Everingham and Rydell, 1994] S.S. Everingham and C.P. Rydell: "Modeling the Demand for Cocaine". MR-332-ONDCP/A/DPRC, RAND: Santa Monica, CA, 1994.

[Greenwood et al., 1998] P.W. Greenwood, K.E. Model, C.P. Rydell, J. Chiesa: Diverting Children from a Life of Crime. MR-699-1-UCB/RC/IF, RAND: Santa Monica, CA, 1998.

[Johnson et al., 1996] R.A. Johnson, D.R. Gerstein, P. Ghadialy, W. Choy, and J. Gfroerer: "Trends in the Incidence of Drug Use in the United States, 1919-1992". Sub- 
stance Abuse and Mental Health Services Administration, Office of Applied Studies, U.S. Department of Health and Human Services, 1996.

[Kaplan, 1983] J. Kaplan: The Hardest Drug: Heroin and Public Policy. University of Chicago Press: Chicago, IL, 1983.

[Kleiman, 1992] M.A.R. Kleiman: Against Excess. Basic Books: New York, NY, 1992.

[Maguire and Pastore, 1996] K. Maguire and A.L. Pastore: Sourcebook of Criminal Justice Statistics 1995. U.S. Department of Justice, Bureau of Justice Statistics: Washington, D.C., 1996.

[Musto, 1973] D.F. Musto: The American Disease. Yale University Press: New Haven, CT, 1973.

[ONDCP, 1998] Office of National Drug Control Policy: The National Drug Control Strategy: 1998: A Ten Year Plan. The White House: Washington, D.C., 1998.

[Rossi and Schinaia, 1998] C. Rossi and G. Schinaia: "The Mover-Stayer Model for the HIV/AIDS Epidemic in Action". Interfaces, 28(3) (1998): 127-143.

[Tobler, 1997] N.S. Tobler: "Meta-Analysis of Adolescent Drug Prevention Programs: Results of the 1993 Meta-Analysis. Meta-Analysis of Drug Abuse Prevention Programs." In NIDA Research Monograph 170, W.J. Bukoski (ed.), U.S. Department of Health and Human Services: Washington, D.C., 1997: 5-68.

[Tragler et al., 2001] G. Tragler, J.P. Caulkins, and G. Feichtinger: "The Impact of Enforcement and Treatment on Illicit Drug Consumption". Operations Research, 49 (2001): 352-362.

[Wolfram, 1996] S. Wolfram: The Mathematica Book, 3rd edition. Wolfram Media / Cambridge University Press, 1996. 